\title{
Efectos adversos derivados de la exposición ocupacional a ozono industrial
}

\section{The adverse effects of occupational exposure to ozone industrial}

\author{
Arias-Hualpa M. Alejandra ${ }^{1,4}$, Leal-Padín M. ${ }^{a}$ Angélica ${ }^{2,4}$, Pérez-Alemán Áurea Isabel ${ }^{3,4}$ \\ 1. Unidad Docente de Medicina del Trabajo de La Rioja. La Rioja. España \\ 2. Unidad Docente de Medicina del Trabajo de Galicia. Galicia. España \\ 3. Unidad Docente de Medicina del Trabajo de Canarias. Canarias. España \\ 4. Unidad Docente de Medicina del Trabajo de la Comunidad de Madrid. Madrid. España
}

Recibido: 04-03-15

Aceptado: 10-03-15

\section{Correspondencia}

Leal Padín, M. Angélica

MIR Medicina del Trabajo. Unidad Docente Galicia

Instituto de Salud Carlos III.

Escuela Nacional de Medicina del Trabajo.

Av. de Monforte de Lemos, 5.

28029 Madrid. España.

Correo electrónico: revisioncusmet@outlook.es

Teléfono: 687923980

Este trabajo se ha desarrollado dentro del Programa Científico de la Escuela Nacional de Medicina del Trabajo del Instituto de Salud Carlos III en convenio con Unidad Docente de Medicina del Trabajo de la Comunidad de Madrid.

Resumen

Introducción: Los trabajadores que realizan tareas de soldadura, depuración de aguas, conservación de alimentos o desempeñan actividades en contacto con fotocopiadoras, lámparas de vapor de mercurio, generadores de rayos $\mathrm{x}$ y otros equipos de alta tensión se pueden encontrar expuestos a ozono, que a dosis altas, puede desencadenar una liberación endógena de mediadores de la inflamación produciendo irritación ocular y daño de la vía respiratoria superior e inferior, pudiendo llegar a producir enfermedad pulmonar obstructiva crónica o edema de pulmón.

Objetivo: Revisar la literatura científica sobre los efectos adversos derivados de la exposición ocupacional a ozono industrial.

Metodología: Se realizó una búsqueda en las principales bases de datos bibliográficas biomédicas, con el fin de recopilar artículos publicados que cumplieran los requisitos del estudio, sin fecha límite inferior, hasta noviembre 2014 .

Resultados: Se incluyeron en el estudio un total de 9 artículos que cumplían los criterios de inclusión. Como hallazgos del estudio se encontró un descenso de la ceruloplasmina como biomarcador de daño oxidativo pulmonar asociado a concentraciones de ozono superiores a los valores límites aceptados, así como aumento del riesgo de rinitis no infecciosa, EPOC leve y síntomas de asma, especialmente las sibilancias.

Conclusión: Los estudios revelan que las dosis altas de ozono industrial pueden representar un riesgo para la salud en determinados grupos de trabajadores como los soldadores y del blanqueado de papel, en los que la patología respiratoria ocupa un papel importante entre los efectos adversos encontrados.

Med Segur Trab (Internet) 2015; 61 (238) 125-138

Palabras clave: Ozono; Exposición ocupacional. 


\section{Abstract}

Introduction: Workers who perform welding tasks, water purification, food preservation or play activities in contact with photocopiers, mercury vapor lamps, x-ray generators or any other high voltage equipments, can be exposed to ozone. At high doses, may trigger a release of endogenous mediators inflammation, causing eye irritation and damage to the upper and lower respiratory tract, being able to produce pulmonary edema or chronic obstructive pulmonary disease.

Objective: To review scientific literature on the adverse effects of occupational exposure to industrial ozone.

Methodology: A search was performed in major biomedical bibliographic data bases in order to collect published articles that met the study requirements, without bottom deadline until November 2014.

Results: A total of 9 articles that met the inclusion criteria were included in the study. As a result of the study, it is found decreased ceruloplasmin as a biomarker of lung oxidative damage associated with concentrations exceeding the accepted limit values ozone and increased risk of non-infectious rhinitis, mild COPD and asthma symptoms, especially wheezing.

Conclusion: Studies revealed that high doses industrial ozone may pose a health risk to certain groups of exposed workers such as welders and paper bleaching, in which the respiratory disease plays an important role among those found adverse effects.

Med Segur Trab (Internet) 2015; 61 (238) 125-138

Key words: Ozone; Occupational Exposure. 


\section{INTRODUCCIÓN}

El descubrimiento, desarrollo y aplicación de nuevos productos químicos, ha permitido mejorar las condiciones de vida de la población, pero al mismo tiempo, ha ocasionado efectos desfavorables para la salud humana. El conocimiento de estos efectos es esencial, para prevenir y controlar los riesgos de los productos químicos. Actualmente, se contabilizan más de 100.000 sustancias químicas, y la mayoría de ellas no han sido controladas desde el punto de vista de evaluación del riesgo ${ }^{1}$.

Se estima que cada año mueren en España 4.000 trabajadores, al menos 33.000 enferman y más de 18.000 sufren accidentes a causa de la exposición a sustancias químicas peligrosas en su trabajo ${ }^{2}$.

Una de las sustancia químicas cada vez más comúnmente utilizada en el ámbito industrial y en el campo de la medicina, es el ozono. Dicho agente se compone de tres átomos de oxígeno y cuya fórmula es $\mathrm{O}_{3}{ }^{3}$.

La exposición ocupacional afecta a profesionales que desempeñan trabajos de soldadura por arco eléctrico, depuración de aguas, conservación de alimentos y tratamiento de cultivos agrícolas, así como los que se encuentran en contacto con lámparas de vapor de mercurio, fotocopiadoras de oficina, generadores de rayos $\mathrm{X}$ y otros equipos eléctricos de alta tensión.

Actualmente la utilización del ozono se ha extendido en medicina como agente terapéutico, bien por su alta capacidad oxidante y de cicatrización o como medio de activación de ciertas proteínas y células del sistema inmune ${ }^{4}$.

A pesar de su extendido uso, algunas fuentes lo definen como un gas tóxico capaz de disminuir la capacidad ventilatoria ${ }^{5}$. Los efectos biológicos se atribuyen a su capacidad para causar oxidación y peroxidación de biomoléculas, directamente y/o por medio de la liberación de radicales libres ${ }^{6-8}$, por lo que es capaz de agotar los recursos antioxidantes el organismo ${ }^{2,6,9}$.

Su exposición desencadena la liberación endógena de mediadores de la inflamación ${ }^{10}$, produciendo irritación ocular y daño de la vía respiratoria superior y daño del epitelio bronquial $^{8}$.

Existe relación entre la dosis recibida de ozono y el grado de lesión epitelial. ${ }^{11}$ Las células alveolares tipo I son susceptibles a la mayoría de los efectos del ozono y pueden sufren necrosis y/o apoptosis, mientras que las de tipo II, son más resistentes y proliferan incluso durante la exposición continua a este gas. ${ }^{12}$ Finalmente, como consecuencia del daño, puede producir enfermedad pulmonar obstructiva crónica (EPOC) y edema de pulmón. ${ }^{8,13,14}$

En animales experimentales el ozono puede aumentar la susceptibilidad a la neumonía bacteriana. Probablemente esto se deba al daño a la membrana de los macrófagos, perjudicando su capacidad para producir el radical anión superóxido que reduce la actividad bactericida. ${ }^{12}$

La legislación, respecto al uso de esta sustancia química en España, está regulada por el Real Decreto 374/2001. ${ }^{15}$ Es clasificada en el Real Decreto 1299/2006 de 10 de noviembre, ${ }^{16}$ según la recomendación 2003/670/CE de la Comisión, del 19 de septiembre de 2003, relativa a la lista europea de enfermedades profesionales, como sustancia del anexo II, Grupo 1 (lista complementaria de enfermedades cuyo origen profesional se sospecha y cuya inclusión en el anexo I podría contemplarse en el futuro). ${ }^{17}$

El límite recomendado de exposición es considerado para trabajo pesado 0,05ppm $\left(0,1 \mathrm{mg} / \mathrm{m}^{3}\right)$, trabajo moderado $0,08 \mathrm{ppm}\left(0,16 \mathrm{mg} / \mathrm{m}^{3}\right)$, trabajo ligero $0,1\left(0,2 \mathrm{mg} / \mathrm{m}^{3}\right)$ calculado como una concentración ponderada de 8 horas. Y trabajo pesado, moderado o ligero (menor o igual a 2 horas) $0,2 \mathrm{ppm}\left(0,4 \mathrm{mg} / \mathrm{m}^{3}\right) .{ }^{18}$ 
En base a todo esto, el objetivo de la presente revisión será revisar la literatura científica sobre los efectos adversos derivados de la exposición ocupacional al ozono industrial y así permitir un mejor conocimiento de los mecanismos de aparición de algunas enfermedades profesionales y su vinculación con dicho agente químico.

\section{METODOLOGÍA}

Se realizó una búsqueda bibliográfica de la literatura científica reciente que abordase los efectos adversos derivados de la exposición ocupacional a ozono industrial mediante consulta en las principales bases de datos biomédicas, MEDLINE-PubMed, Web of Science, LILACS, The Cochrane Library, Scielo y OSH Update.

A partir del Medical Subject Headings (MeSH) se identificaron los principales descriptores del que se relacionaban con el objetivo de la búsqueda: "Occupational Exposure", "Ozone" y "Health Personnel", a partir de los cuales se definieron las siguientes estrategias de búsqueda completada con calificadores:

- MEDLINE/PUBMED:(("Occupational Exposure”[MESH]AND "Ozone/adverse effects "[All Fields])) y ("Health Personnel"[Mesh]) AND (("Ozone/adverse effects"[Mesh] OR "Ozone/toxicity"[Mesh])). Añadiendo los artículos relacionados con estas búsquedas.

- OSH UPDATE: "All Fields Ozone/adverse effects AND occupational Hazards not code Exposure Environment".

— WEB OF SCIENCE: "Ozone/toxicity OR Ozone/adverse effects AND "Occupational Exposure").

La misma ecuación se adaptó al resto de las bases de datos consultadas.

Para su aplicación se emplearon conectores booleanos. Se recopilaron los artículos publicados sin fecha límite inferior de búsqueda, hasta el 30 de noviembre de 2014.

La elección de los artículos, se llevó a cabo mediante el cumplimiento de los siguientes criterios de inclusión:

- Estudios con alta evidencia científica I y II.

- Estudios aplicados en humanos, sin limitación de edad ni sexo.

- Estudios en español, inglés, italiano, portugués, francés y sin límite de fecha.

- Artículos a texto completo.

- Artículos sobre los efectos adversos derivados de la exposición ocupacional a ozono industrial.

- Trabajadores expuestos a ozono industrial.

Se excluyeron los artículos que no aportaban información empírica, sobre los efectos adversos del ozono industrial, en trabajadores expuestos y sobre el ozono ambiental. Se descartaron editoriales, cartas al director, opiniones de expertos, comunicaciones a congresos, a propósito de un caso, estudios aplicados en autopsias, animales de laboratorio y aquellos artículos a los que no pudimos acceder al texto completo.

Para determinar la pertinencia de los artículos, cada uno de ellos se evaluó de forma independiente por dos de los autores. Para dar por válida la elección de los artículos recuperados, se estableció que la valoración de la concordancia entre los revisores, mediante el índice kappa, debía ser superior a 0,6 (fuerza de concordancia buena o muy buena) resultando el índice kappa en $0,90 \mathrm{p}<0,001$. Las discordancias se solucionaron mediante la valoración de un tercer autor de dicha revisión. 


\section{RESULTADOS}

Con los criterios de búsqueda se localizaron un total de 140 artículos, de ellos 68 (48\%) se obtuvieron a partir de MEDLINE-PubMed, 51 (36\%) de la Web of Science, 15 (11\%) de OSH-Update, 4 (3\%) de LILACS, 1 (1\%) de Scielo y 1 (1\%) de Cochrane Library. Tras depurar los artículos duplicados 8 (6\%) y someterse a la revisión de dos autores para evaluar la pertinencia y el cumplimiento de los criterios de inclusión, fueron eliminados $123(87 \%)$ artículos, quedando finalmente 9 (7\%) seleccionados para la revisión. ${ }^{19-27}$

La Tabla I resume los datos más relevantes de cada uno de los artículos referentes a los autores, país donde se realiza el estudio, año de publicación, tipo de estudio, tamaño y tipo de muestra, tiempo de exposición, instrumento de medida, valor ambiental, variable resultado, factores de confusión y nivel de evidencia científica según metodología SIGN (Scottish Intercollegiate Guidelines Network). ${ }^{28}$

De los 9 artículos seleccionados, 7 (78\%) fueron de casos y controles, ${ }^{19-25} 1$ (11\%) cohortes ${ }^{26}$ y un ensayo clínico cuasi-experimental. ${ }^{27}$ Cinco de los estudios fueron realizados en Suecia, ${ }^{20,21,23-25}$ tres de ellos realizados por un mismo autor, dos de los trabajos se realizaron en Polonia, ${ }^{22,27}$ los otros dos artículos restantes fueron realizados en Francia ${ }^{19}$ y USA. ${ }^{26}$ El tamaño de las muestras, osciló entre, $\mathrm{n}=12$ y n= 325. (Tabla 1).

Pierre F. et al. ${ }^{19}$ evaluó los efectos de la exposición continua a ozono en trabajadores soldadores, a través de la ceruloplasmina como biomarcador de daño oxidativo pulmonar, evidenciando disminución de esta en el grupo de expuestos $(\mathrm{p}=0,01)$.

A.C Olin et al. realizó tres estudios en diferentes años. ${ }^{20,21,23}$ Uno de ellos, realizado en 1999, estudia los efectos en trabajadores del blanqueado de papel. En este se evaluó el óxido nítrico exhalado, no observando relación entre los controles y los sujetos expuestos a elevaciones continuas de ozono (media de 67,2nL.min-1 frente a 55,0nL.min1). Sin embargo si se evidenció diferencia entre los expuestos a concentraciones pico ocasional. La media de salida del óxido nítrico fue de 90,0nL.min-1 en expuestos, frente a 58.8nL.min-1 en no expuestos $(\mathrm{p}=0.016){ }^{20}$

Otro de los estudios realizados por el mismo autor en $2002^{21}$, detecta un aumento de la incidencia de la sibilancias (tasa de incidencia de 2,3 con un de IC al 95\% de 1.6 a 5,8). Este mismo autor en un estudio realizado en $2004^{23}$ no observó diferencias en la función pulmonar en los expuestos (FEV1 del 99,9\%) frente (105,3\%) a los no expuestos $(\mathrm{p}=0.17)$. Tampoco se encontró diferencias entre la media de concentración de óxido nítrico exhalado entre casos y controles (15.9 ppb frente a16.9 ppb; $\mathrm{p}=0.32$ ). A pesar de ello, los trabajadores expuestos a mayores concentraciones, presentaron una media de óxido nítrico mayor que los expuestos a menos cantidades (19.2 vs 15.7 ppb, p=0.04).

Stepnewski M. et $\mathbf{a l}^{22}$, en su estudio, observa que casi el doble de los soldadores expuestos estaban en riesgo de EPOC leve $(\mathrm{p}<0.001)$ aunque a pesar de observar síntomas respiratorios moderados en expuestos $(8,5 \%)$ versus controles $(0.9 \%)$ no hubo diferencias $(\mathrm{p}<0.02)$. Por otro lado se señala que el $32 \%$ de los soldadores, presentaban obstrucción de la vía aérea, mientras que en los controles tal hallazgo clínico se observó en un $20 \%$ IC $95 \%(\mathrm{p}<0.05)$. 


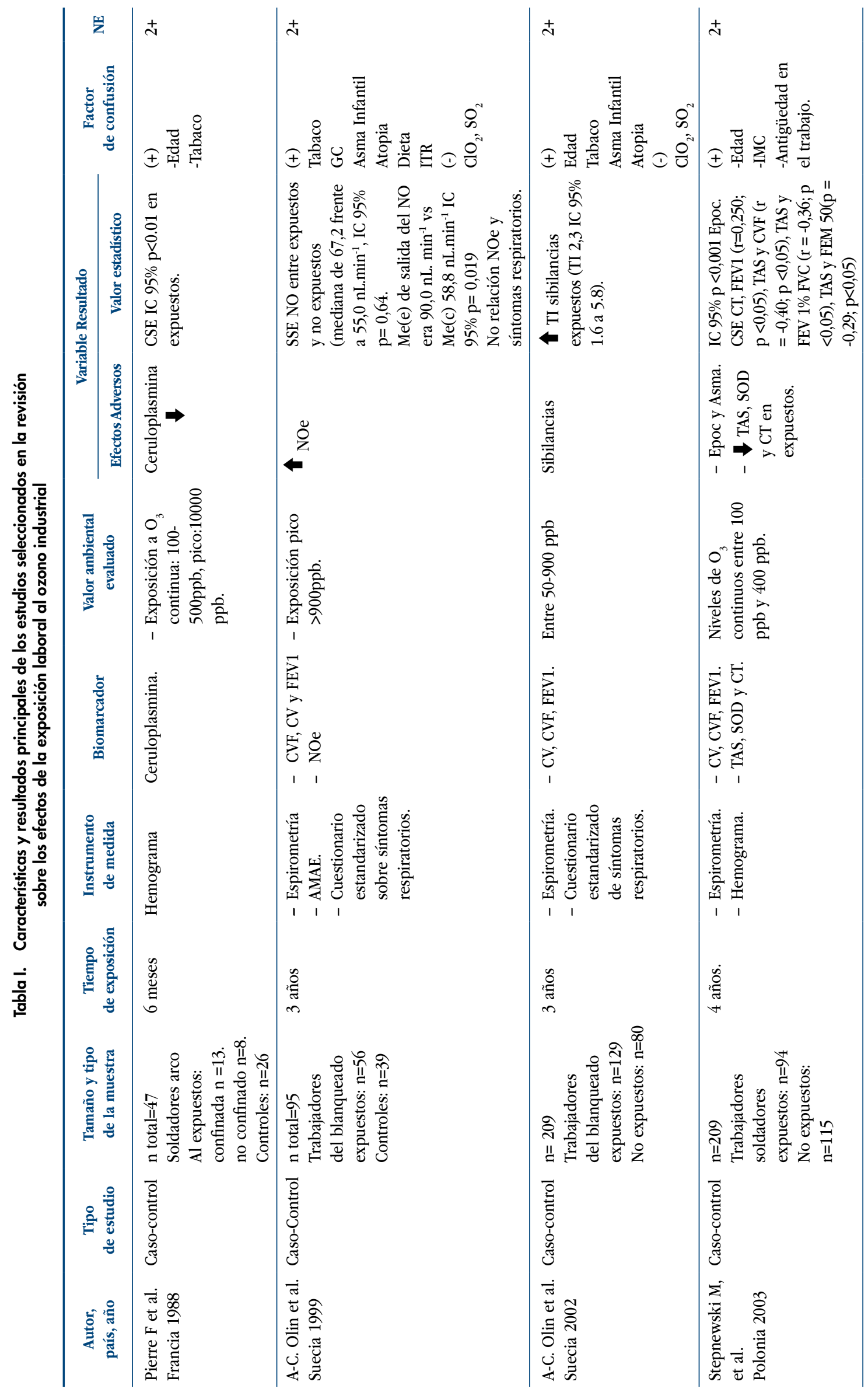




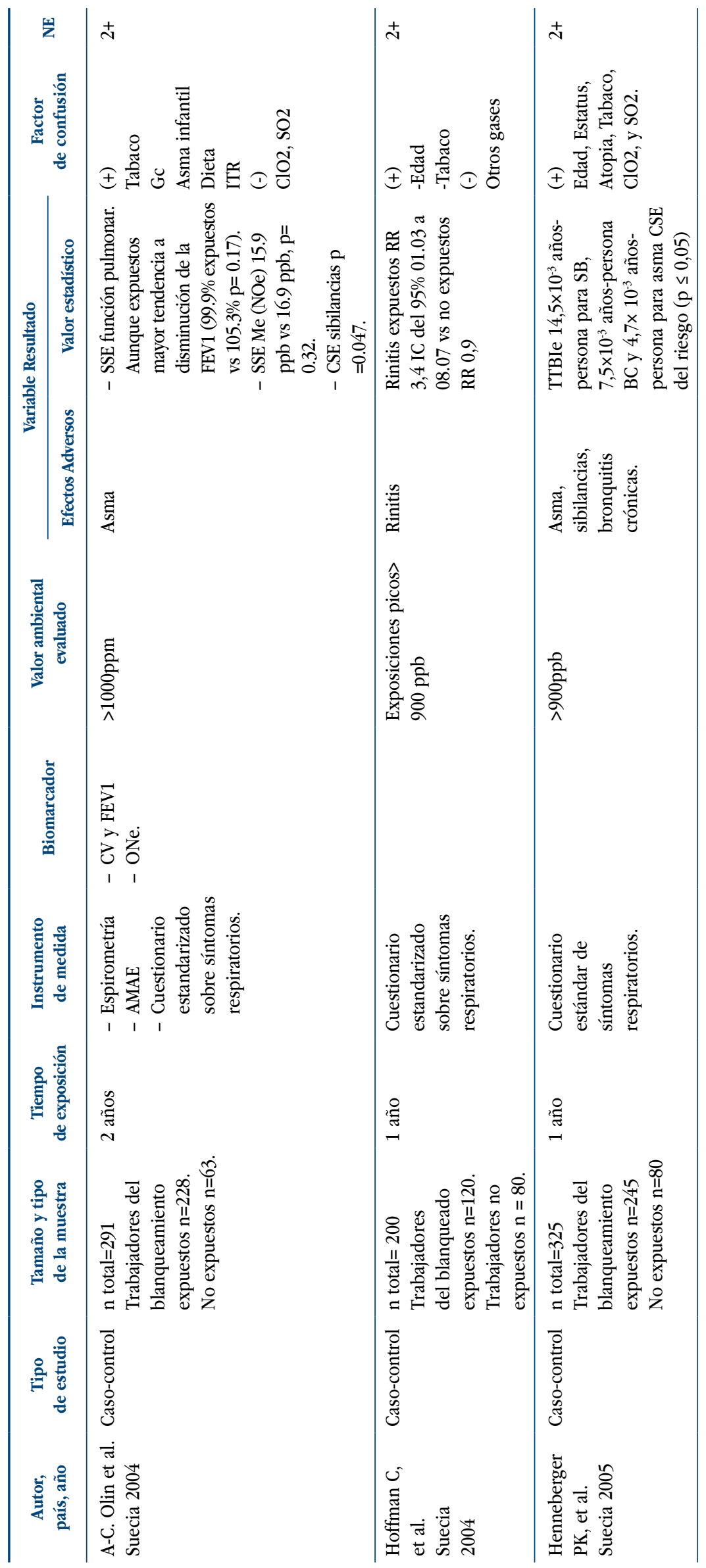




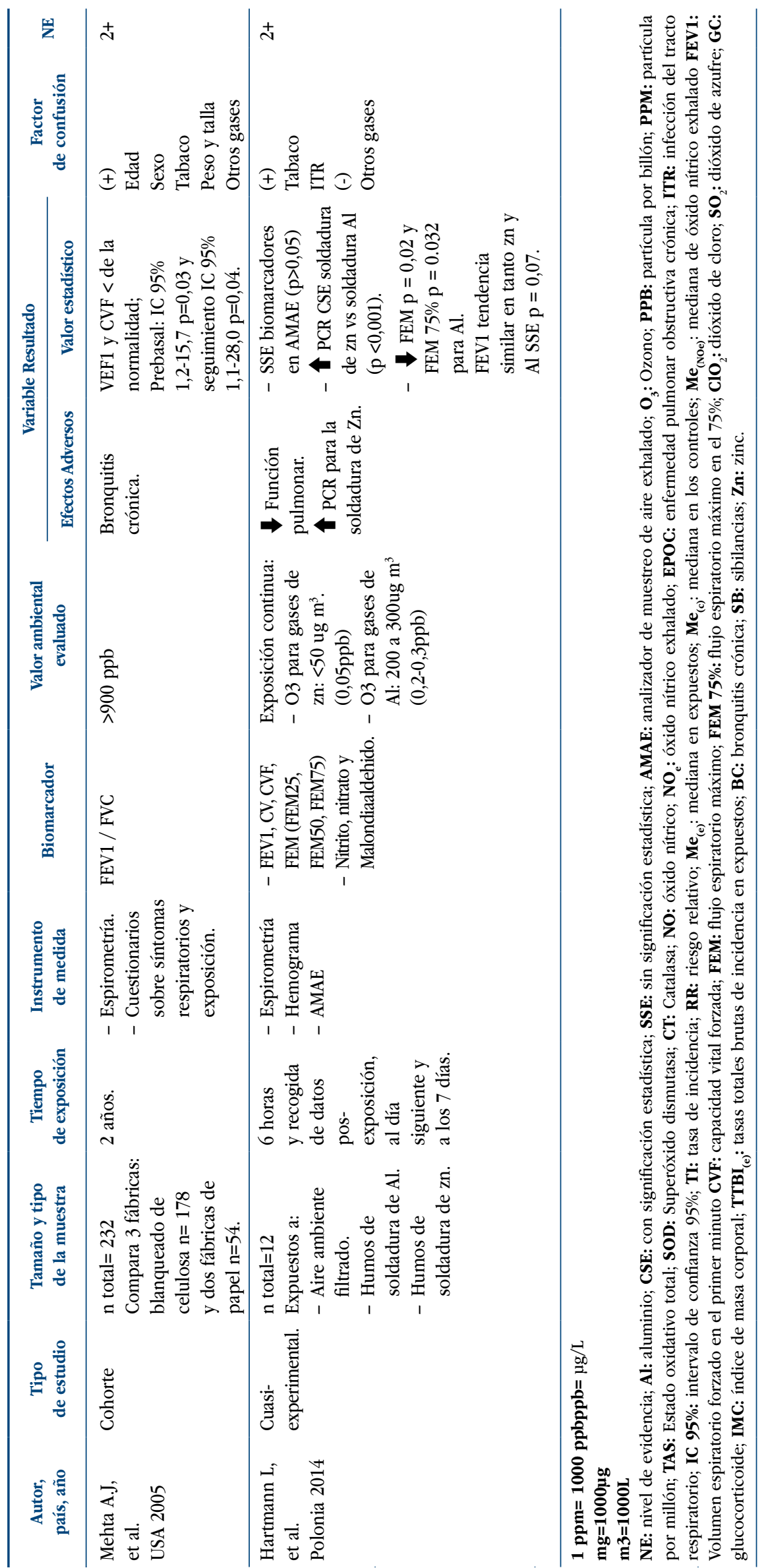


Se determinó el estado antioxidante mediante el estado oxidativo total, superóxido dismutasa y catalasa. El análisis estadístico no reveló diferencias significativas, aunque la actividad de la catalasa en el grupo de soldadores fue mucho menor (aproximadamente 36\%) que en los controles. Esta diferencia, sin embargo, no fue estadísticamente significativa $(p<0,08)$.

También se evidenció correlación significativa entre CT y FEV1 ( $r=-0,250 ; p<0,05)$, y también entre TAS y FVC $(r=-0,40 ; p<0,05)$, TAS y FEV1\% FVC $(r=-0.36 ; p<0,05)$, TAS y MEF50 (p = - 0,29; p <0,05). ${ }^{22}$

Hoffman C. et al. ${ }^{24}$ observó un aumento del riesgo de rinitis no infecciosa $\mathrm{RR}=3,4$ $\mathrm{IC}=95 \% ; 1,03$ a 8,07 en expuestos frente a no expuestos $(\mathrm{RR}=0,9)$.

Por su parte, Henneberger PK, et al. ${ }^{25}$ evidencia que las tasas totales brutas de incidencia en expuestos, para sibilancias 14,5×10-3 año-persona, bronquitis crónica $7,5 \times 10-3$ año-persona y para el asma 4,7x10-3 año-persona, se asoció con un aumento de riesgo de padecer dichas patologías $(\mathrm{p}<0,05)$.

El estudio de Metha A.J et al. ${ }^{26}$ definió la limitación crónica al flujo aéreo como la FEV1/CVF por debajo de la normalidad. Dicho autor obtuvo como resultados en la espirometría postexposición (IC95\% 1,1-28 p=0,04).

Por último, Hartmann $L$ et al. ${ }^{27}$ realiza un estudio cuasi experimental, en el que un grupo de trabajadores fue expuesto a aire ambiente filtrado, humos de soldadura de aluminio y soldadura de zinc, con periodo de lavado de 7 días entre cada exposición. Observando elevación del PCR durante la exposición a los gases de soldadura de zinc en comparación con la soldadura de aluminio $(\mathrm{p}<0,001)$, y disminución del FEM en la soldadura del aluminio $(\mathrm{p}=0,02)$ y $\operatorname{FEM} 75 \%(\mathrm{p}=0,032)$.

En las siguientes gráficas se observan las concentraciones pico ocasional (gráfica 1) y las concentraciones continúas de ozono medidas por los autores (gráfica 2).

Gráfica 1. Concentración ambiental de corta duración detectada (exposición pico)

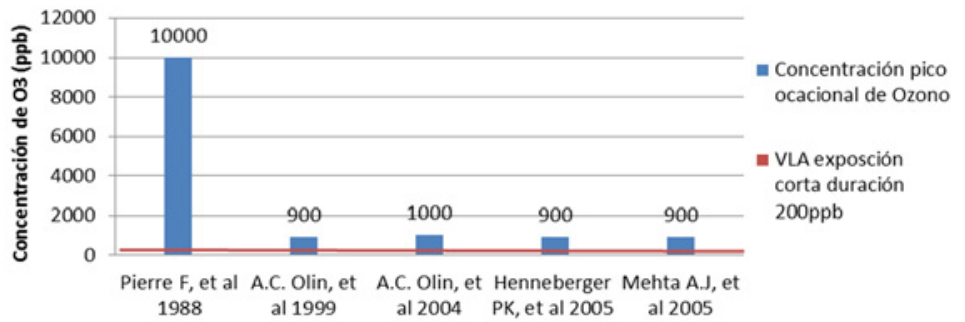

Se observa en la gráfica 1, las concentraciones pico (referida por los autores como las elevaciones de ozono producidas por escapes accidentales en el puesto de trabajo). Se evidencia que la mayoría detectó elevaciones por encima de los valores límites ambientales establecidos ${ }^{18}$, equivalente a $200 \mathrm{ppb}$.

Gráfica 2. Concentración ambiental mínima y máxima detectada

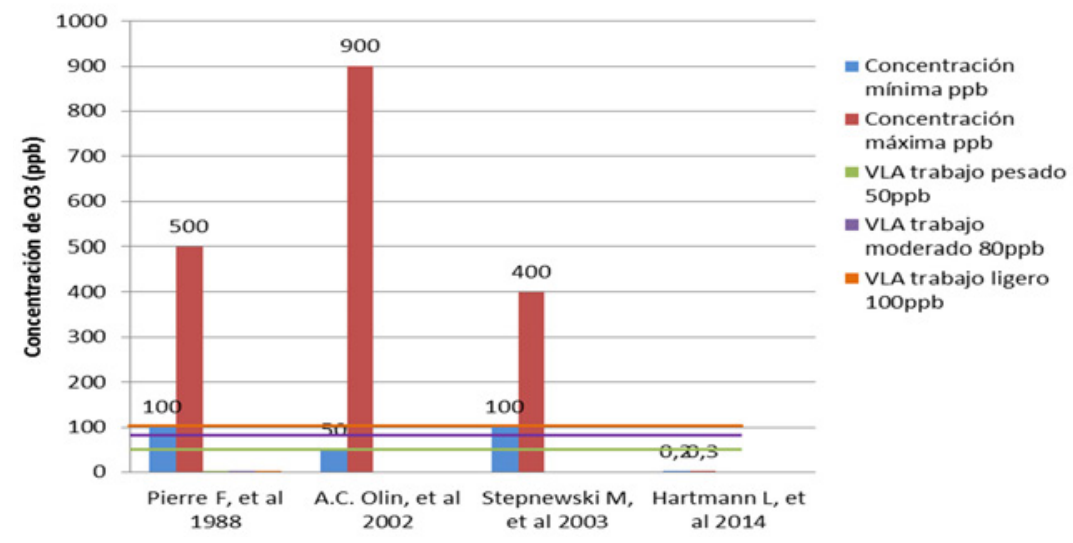

Efectos adversos derivados de la exposición ocupacional a ozono industrial Arias-Hualpa M. Alejandra, Leal-Padín M. ${ }^{a}$ Angélica, Pérez-Alemán Áurea Isabel 
Puede observarse en la gráfica 2, las concentraciones continuas máximas y mínimas evidenciadas para los diferentes estudios. ${ }^{19,20,22,27}$ Cabe destacar que los autores no especifican si la actividad realizada por los trabajadores era de tipo pesado, moderado o ligero, por lo que se comparan en la gráfica los tres valores que establece la legislación. ${ }^{18}$ Tres de los autores ${ }^{19,20,22}$ detectan que las concentraciones mínimas y máximas se encontraban por encima de los valores límites ambientales (para las tres categorías de trabajo, pesado, moderado y ligero). Se menciona que las concentraciones de ozono medida por uno de los autores ${ }^{27}$ fueron mínimas, encontrándose dentro de los valores límites ambientales establecidos.

\section{DISCUSIÓN}

La mayor parte de los autores estudiaron la exposición de ozono en trabajadores del blanqueado de papel $^{20,21,23-26}$ y en menor cuantía la exposición en trabajadores soldadores. ${ }^{19,22,27}$ Además evaluaron el valor ambiental continuo de ozono ${ }^{19-27}$ y elevaciones puntuales accidentales producidas por escapes. ${ }^{19,20,23,25,26}$

Los principales aspectos que se estudiaron fueron los siguientes:

\section{Clínica}

Stepnewski et al. ${ }^{22}$ observa que en los soldadores expuestos, casi el doble estaban en riesgo de enfermedad pulmonar obstructiva crónica $(\mathrm{p}<0,001)$ en comparación con los controles. En la misma proporción aunque el análisis estadístico demostró que no hubo diferencia, se encontraron síntomas moderados de EPOC en 8 soldadores $(8,5 \%)$, pero sólo en un sujeto $(0,9 \%)$ del grupo control.

Se encuentran algunas publicaciones que informan alteraciones del tracto respiratorio relacionado con la exposición a gases oxidantes y vapores generados durante la soldadura $^{29,30}$ y que corroboran que los trabajadores soldadores son propensos a presentar alteraciones respiratorias de tipo obstructivo. ${ }^{31,32}$

Otros estudios seleccionados evidencian que los trabajadores del blanqueado de papel que han estado expuestos repetidamente a picos elevados de ozono, tienen una mayor prevalencia de asma, incrementándose la incidencia de sibilancia. ${ }^{21,23,25}$ Además concluyen que estos trabajadores expuestos tienen mayor prevalencia de bronquitis crónica evidenciando patrón obstructivo. ${ }^{25}$

A pesar de estos resultados, Olin et al. ${ }^{20,21,23}$ en sus tres estudios, no se pudo determinar si el aumento del riesgo de síntomas respiratorios fue debido al ozono u a otros gases como el dióxido de cloro y dióxido de azufre. Esto pudo deberse a que en los procesos del blanqueado también se liberan estos gases oxidantes.

Hoffman et al. ${ }^{24}$ observó que la exposición aguda a altos niveles de ozono aumenta el riesgo de la rinitis no infecciosa en los trabajadores del blanqueado. Sin embargo, el riesgo de aparición de sintomatología respiratoria es valorado a través de cuestionarios estandarizados. ${ }^{20,21,23,24,26}$ El uso de este método en vez de medidas más objetivas, pudo haber ocasionado subestimación el riesgo de rinitis u otros síntomas a esta población de estudio. Por último, las personas que presentaban síntomas en el momento de la aplicación del cuestionario pudieron ser más propensos a dar respuestas afirmativas que aquellos cuyos síntomas se habían resuelto antes de la administración del cuestionario.

El dióxido de cloro también puede haber actuado como modificador del efecto. Este agente fue usado en los procesos de blanqueado de papel antes de la inserción del ozono. El riesgo de desarrollar síntomas respiratorios como consecuencia del ozono quizás fue mayor entre los trabajadores previamente expuestos a dióxido de cloro, ya que la respuesta inflamatoria debido a picos de cloro repetidos pudo no haber desaparecido por completo. 
Por tanto, el dióxido de cloro y otro gas como lo es el dióxido de azufre, se presentan como factores de confusión por lo que pudieron ser los causantes de los síntomas respiratorios evidenciados..$^{20,21,23,24,27}$

Estudios demuestran que los ingresos hospitalarios de pacientes con asma, aumentan cuando las concentraciones ambientales de ozono están elevadas ( $>100 \mathrm{ppb}) .{ }^{33}$ Además también se sabe que la exposición a irritantes, puede provocar hiperreactividad bronquial, de tal manera que estos forman parte del mecanismo causal para desarrollar asma. ${ }^{34,35}$

También se ha descrito, que los trabajadores del blanqueado, tras la inhalación a altas concentraciones de ozono, presentan daño del epitelio de las vías respiratorias superiores, provocando inflamación, alteraciones de la función pulmonar ${ }^{13,36}$ y como consecuencia síntomas respiratorios como dolor de garganta, tos, disnea y dolor torácico, ${ }^{14,37,38}$ así como enfermedad pulmonar obstructiva crónica y otras diversas enfermedades de las vías respiratorias. ${ }^{39,40}$

\section{Estados oxidativos}

Algunos autores determinaron el daño en el equilibrio oxidativo a partir de marcadores bioquímicos. El propósito principal de estos estudios fue investigar los parámetros ventilatorios en relación con el estado antioxidante, medido como el estado antioxidante total, superóxido dismutasa y catalasa, ${ }^{22}$ así como el óxido nítrico en el aire exhalado. $^{20,23}$

Stepnewski et al. ${ }^{22}$ observan que el estado antioxidante total (TAS) en los soldadores expuestos se encontraba por debajo de la normalidad $(\mathrm{p}<0,001)$. Del mismo modo se evidenció para la catalasa (CT) y superóxido dismutasa (SOD) respectivamente $(\mathrm{p}<0.01)$.

Sin embargo, a pesar de estos resultados, la búsqueda de una correlación entre los parámetros espirométricos y la amplia gama de enzimas sanguíneas y antioxidantes fueron insatisfactorias. Por tanto es evidente que los cambios en la concentración de estos parámetros no deben ser utilizados como marcadores tempranos de la disfunción ventilatoria.

Los niveles de óxido nítrico en el aire exhalado se han propuesto como un biomarcador de la inflamación de las vías respiratorias. ${ }^{41} \mathrm{Su}$ elevación se traduce en inflamación eosinófila, es decir, en la relacionada con la atopia aunque no es exclusiva de esta.

Olin et al. ${ }^{20}$ indican que las concentraciones de óxido nítrico en el aire exhalado se incrementa entre los trabajadores expuestos a altos niveles de ozono y dióxido de cloro, lo que podría suponer que la exposición a ozono causa inflamación de las vías respiratorias y aumento concomitante en la salida de óxido nítrico. A pesar de ello la elevación del óxido nítrico exhalado no se relacionó con mayor presentación de síntomas respiratorios o cambios en la espirometría.

La principal limitación de estos estudios es que el método utilizado para la medición del óxido nítrico permite la contaminación del aire exhalado por el aire procedente de las vía respiratoria superior, ${ }^{42}$ lo que podría dar como resultado falsas elevaciones del óxido nítrico.

Olin et al. ${ }^{20,23}$ corrigen ciertos factores de confusión tales como la dieta, ${ }^{43}$ infecciones virales, ${ }^{44}$ uso de glucocorticoides y asma desde la infancia, ${ }^{45}$ ya que el óxido nítrico exhalado puede elevarse en otras situaciones.

Pierre et al. ${ }^{19}$ utilizan la ceruloplasmina como marcador de estrés oxidativo, detectando una disminución significativa del valor biológico en los soldadores expuestos a ozono en atmósfera confinada $(\mathrm{p}<0.01)$.

La ceruloplasmina ha resultado ser protectora contra la lesión oxidante de los pulmones, ${ }^{46}$ sin embargo, la actividad oxidasa de la ceruloplasmina es inespecífica y participa en reacciones de oxidación de diversos sustratos. ${ }^{47}$ 


\section{Espirometría}

Se revisaron estudios donde los trabajadores expuestos a niveles altos de ozono tenían FEV1 significativamente menor que los controles, sin embargo no hubo diferencia significativa con respecto a la FVC $(\mathrm{p}=0.007){ }^{20}$ Olin et al. ${ }^{23}$ también observan que no existían diferencias en la función pulmonar entre trabajadores del blanqueado, aunque los trabajadores con mayor exposición tenían tendencia a disminuir FEV.

Mehta et al. ${ }^{26}$ encontraron que la tasa de disminución del FEV1/FVC fue mayor para los trabajadores expuestos a altas concentraciones de ozono, aunque no hubo diferencias significativas $(\mathrm{p}=0.19)$.

El hallazgo más reciente lo encontramos en un estudio realizado por Hartmann et al. ${ }^{27}$ en el cual se observó una disminución significativa de FEM ( $\left.\mathrm{p}=0,07\right)$ y FEM75\% $(\mathrm{p}=0,032)$ después de la exposición a humos de soldadura de aluminio $(\mathrm{p}=0,047)$, esto se tradujo en una disminución ligera de la función pulmonar que pudo atribuirse a la exposición del ozono. La FEV1 mostró una tendencia a la disminución para ambos gases sin evidencia estadística. ${ }^{27}$

Se han encontrado otros estudios, donde se describe también una disminución FEV1/FVC en los trabajadores expuestos al ozono inhalado ${ }^{39}$.

En general, todos los grupos de expuestos, presentaron parámetros espirométricos por debajo de los valores normales, en comparación con el grupo control. Sin embargo en los resultados obtenidos no se evidenciaron alteraciones graves de la función pulmonar. Esto pudo ser atribuido, a que tuviera que transcurrir mayor tiempo de exposición, para provocar cambios mayores en el árbol bronquial y ocasionar mayores síntomas asociados y/o decrementos espirométricos de tipo obstructivo. Además de ello aquellos trabajadores que presentaron síntomas respiratorios o cambios espirométricos durante el seguimiento, fueron separados de sus puestos de trabajo a espacios libres de gases tóxicos y humos. Probablemente esta es la razón que explique la ausencia de EPOC en estadios graves o muy graves u otros síntomas respiratorios severos.

En base a los resultados en los diferentes estudios revisados, es difícil fijar en términos cuantitativos la relación entre la exposición al ozono y los problemas de salud derivados. En su mayoría el nivel de evidencia que fue $2+.{ }^{28}$ Probablemente la evidencia disponible sea la mejor posible dadas las dificultades de estudio en relación a esta sustancia química tan poco investigada en ámbito laboral, llevándonos a establecer conclusiones sobre algunos grupos poblacionales de trabajadores que presentan mayor riesgo ante la exposición.

Los hallazgos sugieren que las exposiciones máximas a irritantes plantean un riesgo de patología respiratoria entre los trabajadores del blanqueado y soldadores. En particular, se observó aumento de riesgo de asma, sibilancias, enfermedad pulmonar obstructiva crónica, rinitis y bronquitis crónica asociada con exposiciones continuas.

En base a esto, se necesitan esfuerzos adicionales para prevenir eventos de alta exposición en trabajadores expuestos, debiéndose aplicar las medidas preventivas adecuadas, con el fin de minimizar dicha exposición y por consiguiente disminuir la incidencia de las patologías derivadas de la misma.

Se recomienda realizar estudios adicionales, abarcando otros grupos de trabajadores potencialmente expuestos, entre ellos los profesionales de la salud. 


\section{REFERENCIAS BIBLIOGRÁFICAS}

1. Ministerio de Sanidad, Servicios Sociales e Igualdad - Ciudadanos - Productos químicos. www.msssi.gob.es/ ciudadanos/saludAmbLaboral/prodQuimicos/introduccion.htm

2. García AM, Gadea R. Estimaciones de incidencia y prevalencia de enfermedades de origen laboral en España. Aten Primaria. 2008;40(9):439-45.

3. Kuchel PW, Ralston GB. Bioquímica General. México: McGraw-Hill; 1997.

4. Viebahn-Hänsler, R.The Use of Ozone in Medicine. ODREI. Publishers, 5th English edition, 176pp. (2007) ISBN 978-3-934181-02-1.

5. Yang Q, Chen Y, Shi Y, Burnett RT, McGrail KM, Krewski D. Association Between Ozone and Respiratory Admissions Among Children and the Elderly in Vancouver, Canada. Inhal Toxicol. 1 de enero de 2003;15(13):1297-308.

6. Pryor WA. Mechanisms of radical formation from reactions of ozone with target molecules in the lung. Free Radic Biol Med. noviembre de 1994;17(5):451-65.

7. Abdrashitova NF, Baliakin I. Comparative study of ion homeostasis and lipid peroxidation in human serum during prolonged exposure to ozone. Aviakosmicheskaia Ekol Meditsina Aerosp Environ Med. 1999;34(6):38-40.

8. Parks S, Paul DW. Ozone exposure: a case report and discussion. J Okla State Med Assoc. 2000;93(2):4851.

9. O'Neill CA, van der Vliet A, Eiserich JP, Last JA, Halliwell B, Cross CE. Oxidative damage by ozone and nitrogen dioxide: synergistic toxicity in vivo but no evidence of synergistic oxidative damage in an extracellular fluid. Biochem Soc Symp. 1995;61:139-52

10. Pryor WA, Squadrito GL, Friedman M. The cascade mechanism to explain ozone toxicity: The role of lipid ozonation products. Free Radic Biol Med. diciembre de 1995;19(6):935-41.

11. Plopper CG, Chow CK, Dungworth DL, Brummer M, Nemeth TJ. Effect of low level of ozone on rat lungs: II. Morphological responses during recovery and re-exposure. Exp Mol Pathol. 1978;29(3):400-11.

12. Waldron. Non-neoplastic Disorders due to Metallic, Chemical and Physical Agents. Third Edition. Oxford, England: Butterworth-Heinemann Ltd; 1994. 593-643 p.MacNee W. Oxidants/antioxidants and COPD. CHEST J. 2000;117(5_suppl_1):303S - 317S.

13. MacNee W. Oxidants/antioxidants and COPD. CHEST J. 2000;117(5_suppl_1):303S -317S.

14. Krishna MT, Mudway I, Kelly FJ, Frew AJ, Holgate ST. Ozone, airways and allergic airways disease. Clin Exp Allergy. 1995;25(12):1150-8.

15. Real Decreto $374 / 2001$, de 6 de abril sobre la protección de la salud y seguridad de los trabajadores contra los riesgos relacionados con los agentes químicos durante el trabajo. Instituto Nacional de Seguridad e Higiene en el Trabajo (INSHT). Boletín Oficial del Estado, $\mathrm{n}^{\circ} 104$ (01-05-2001).

16. Real Decreto 1299/2006, de 10 de noviembre, por el que se aprueba el cuadro de enfermedades profesionales en el sistema de la Seguridad Social y se establecen criterios para su notificación y registro. Instituto Nacional de Seguridad e Higiene en el Trabajo (INSHT). Boletín oficial del estado n $^{\circ} 302$ (19-122006).

17. Recomendación 2003/670/CE de la Comisión, de 19 de septiembre de 2003, relativa a la lista europea de enfermedades profesionales. Instituto Nacional de Seguridad e Higiene en el Trabajo (INSHT).

18. Instituto Nacional de Seguridad e Higiene en el Trabajo. Límites de Exposición Profesional para agentes químicos en España. Madrid. 2014.

19. Pierre F, Baruthio F, Diebold F, Wild P, Goutet M. Decreased serum ceruloplasmin concentration in aluminum welders exposed to ozone. Int Arch Occup Environ Health. 1988;60(2):95-7.

20. Olin A-C, Ljungkvist G, Bake B, Hagberg S, Henriksson L, Toren K. Exhaled nitric oxide among pulpmill workers reporting gassing incidents involving ozone and chlorine dioxide. Eur Respir J. 1999;14(4):828-31.

21. Olin A-C, Granung G, Hagberg S, Adriansson M, Brisman J, Dalander O, et al. Respiratory health among bleachery workers exposed to ozone and chlorine dioxide. Scand J Work Environ Health. abril de $2002 ; 28(2): 117-23$.

22. Stepnewski M, Kolarzyk E, Pietrzycka A, Kitlinski M, Helbin J, Brzyszczan K. Antioxidant enzymes and pulmonary function in steel mill welders. Int J Occup Med Environ Health. 2003;16(1):41-7.

23. Olin A-C, Andersson E, Andersson M, Granung G, Hagberg S, Torén K. Prevalence of asthma and exhaled nitric oxide are increased in bleachery workers exposed to ozone. Eur Respir J. 2004;23(1):87-92.

24. Hoffman CD, Henneberger PK, Olin A-C, Mehta A, Torén K. Exposure to ozone gases in pulp mills and the onset of rhinitis. Scand J Work Environ Health. diciembre de 2004;30(6):445-9. 
25. Henneberger PK, Olin A-C, Andersson E, Hagberg S, Torén K. The incidence of respiratory symptoms and diseases among pulp mill workers with peak exposures to ozone and other irritant gases. CHEST J 2005;128(4):3028-37.

26. Mehta AJ, Henneberger PK, Torén K, Olin A-C. Airflow limitation and changes in pulmonary function among bleachery workers. Eur Respir J. julio de 2005;26(1):133-9.

27. Hartmann L, Bauer M, Bertram J, Gube M, Lenz K, Reisgen U, et al. Assessment of the biological effects of welding fumes emitted from metal inert gas welding processes of aluminium and zinc-plated materials in humans. Int J Hyg Environ Health. marzo de 2014;217(2-3):160-8.

28. Scottish Intercollegiate Guidelines Network. A guideline developers' handbook (Publication $\mathrm{n}^{\circ}$ 50) Edinburgh: SIGN: 2001 [actualizado mayo de 2004; consultada 18 de junio de 2007]. Disponible en: http://www.sign.ac.uk/guidelines/fulltext/50/index.htm

29. OJIMA J, SHIBATA N, Iwasaki T. Laboratory evaluation of welder's exposure and efficiency of air duct ventilation for welding work in a confined space. Ind Health. 2000;38(1):24-9.

30. Sobaszek A, Edme JL, Boulenguez C, Shirali P, Mereau M, Robin H, et al. Respiratory symptoms and pulmonary function among stainless steel welders. J Occup Environ Med. 1998;40(3):223-9.

31. Nakadate T, Aizawa Y, Yagami T, Zheg Y-Q, Kotani M, Ishiwata K. Change in obstructive pulmonary function as a result of cumulative exposure to welding fumes as determined by magnetopneumography in Japanese arc welders. Occup Environ Med. 1998;55(10):673-7.

32. Wolf C, Pirich C, Waldhoer T, Vallic E. Pulmonary function and symptoms of welders. Int Arch Occup Environ Health. 1997;69(5):350-3.

33. Defense H. Health effects of outdoor air pollution. Am J Respir Crit Care Med. 1996;153:477-98.

34. Tarlo SM. Workplace respiratory irritants and asthma. Occup Med Phila Pa. 1999;15(2):471-84.

35. Horstman DH, Folinsbee LJ, Ives PJ, Abdul-Salaam S, McDonnell WF. Ozone Concentration and Pulmonary Response Relationships for 6.6-Hour Exposures with Five Hours of Moderate Exercise to 0.08, 0.10, and 0.12 ppm. Am Rev Respir Dis. 1 de noviembre de 1990;142(5):1158-63.

36. Folinsbee LJ, McDonnell WF, Horstman DH. Pulmonary function and symptom responses after 6.6-hour exposure to 0.12 ppm ozone with moderate exercise. Japca. 1988;38(1):28-35.

37. Kleinfeld M, Giel CP. Clinical manifestations of ozone poisoning: report of a new source of exposure. Am J Med Sci. 1956;231(6):638-43.

38. Graham DE, Koren HS. Biomarkers of inflammation in ozone-exposed humans: comparison of the nasal and bronchoalveolar lavage. Am Rev Respir Dis. 1990;142(1):152-6.

39. Kennedy SM, Enarson DA, Janssen RG, Chan-Yeung M. Lung health consequences of reported accidental chlorine gas exposures among pulpmill workers. Am J Respir Crit Care Med. 1991;143(1):74-9.

40. Toren K, Hagberg S, Westberg $\mathrm{H}$ akan. Health effects of working in pulp and paper mills: exposure, obstructive airways diseases, hypersensitivity reactions, and cardiovascular diseases. Am J Ind Med. 1996;29(2):111-22.

41. Barnes PJ, Kharitonov SA. Exhaled nitric oxide: a new lung function test. Thorax. 1996;51(3):233-7.

42. Kimberly B, Nejadnik B, Giraud GD, Holden WE. Nasal contribution to exhaled nitric oxide at rest and during breathholding in humans. Am J Respir Crit Care Med. 1996;153(2):829-36.

43. Olin A-C, Aldenbratt A, Ekman A, Ljungkvist G, Jungersten L, Alving K, et al. Increased nitric oxide in exhaled air after intake of a nitrate-rich meal. Respir Med. 2001;95(2):153-8.

44. De Gouw HW, Grunberg K, Schot R, Kroes AC, Dick EC, Sterk PJ. Relationship between exhaled nitric oxide and airway hyperresponsiveness following experimental rhinovirus infection in asthmatic subjects. Eur Respir J. 1998;11(1):126-32.

45. Alving K, Weitzberg E, Lundberg JM. Increased amount of nitric oxide in exhaled air of asthmatics. Eur Respir J. 1993;6(9):1368-70.

46. Galdston M, Levytska V, Schwartz MS, Magnusson B. Ceruloplasmin. Increased serum concentration and impaired antioxidant activity in cigarette smokers, and ability to prevent suppression of elastase inhibitory capacity of alpha 1-proteinase inhibitor. Am Rev Respir Dis. 1984;129(2):258-63.

47. Yapur, V.M., Bustos, M.F., González, A.S., and Negri, G.A. Ceruloplasmina: Determinación de su actividad ferroxidasa. Acta Bioquím ClínLatinoam. 2007; 41: 347-351. 